\title{
Mon dernier coup de pinceau
}

\author{
Myrella Roy, directrice générale de 2003 à 2018
}

L 'année 2018 a marqué la fin de mon mandat à titre de cadre supérieur de la Société canadienne des pharmaciens d'hôpitaux (SCPH) après plus de 15 merveilleuses années gratifiantes. Au moment où vous lirez ces lignes, je me serai engagée dans le chapitre de ma vie consacré à la retraite. J'aimerais profiter de ce rapport pour m'imaginer une promenade avec vous dans la vaste galerie exposant les succès accomplis par la SCPH en 2018 et au cours des 15 dernières années.

Le comité directeur du programme Excellence en pharmacie hospitalière, le chef-d'œuvre, s'est inspiré des réponses aux sondages réalisés à la fin 2017 auprès de patients et de membres de la SCPH. Ces sondages demandaient d'expertiser comment les trois thèmes du programme - les soins centrés sur le patient, les meilleures pratiques, et la communication et la collaboration se traduisent dans les soins prodigués aux patients par les équipes de pharmacies hospitalières. Selon les réponses aux sondages, des cibles réalistes et ambitieuses ont été établies afin d'évaluer le rendement des soins exprimés par les thèmes centraux. Le rapport d'enquêtes (intitulé What Patients and Members Told Us about Patient Care), les cibles du programme et les numéros de la nouvelle infolettre, Excellence Express, sont disponibles au https:// www.cshp.ca/excellence.

Grâce aux concours des muses de l'organisme (ses membres), la SCPH a pris part au mouvement Choisir avec soin Canada (https://choisiravecsoin.org/recommandations/). Six recommandations ont été formulées, incitant les pharmaciens qui exercent dans les hôpitaux et les autres milieux de soins de santé misant sur la collaboration à réduire les pharmacothérapies couramment utilisées mais superflues qui ne sont pas appuyées sur des données probantes et qui pourraient être nuisibles aux patients.

La SCPH et l'Association des pharmaciens des établissements de santé du Québec (A.P.E.S.) ont créé une œuvre d'art collective : une alliance stratégique entièrement mise à jour qui représente une occasion intéressante à saisir pour les membres des deux organismes. Les membres actuels de la SCPH peuvent maintenant jouir des mêmes avantages et services dont profitent les membres associés de l'A.P.E.S., et ce, sans frais supplémentaires. De même, la SCPH offre aux membres actifs de l'A.P.E.S. qui le désirent les mêmes droits et avantages qu'elle accorde à ses partisans individuels, et ce, sans frais supplémentaires. À la faveur de leur alliance stratégique remaniée, la SCPH et l'A.P.E.S. s'efforcent aussi d'accentuer leur voix collective partout au Canada - comme porte-paroles des pharmaciens qui se consacrent à améliorer la santé et la qualité de vie des Canadiens dans les hôpitaux et les autres milieux de soins de santé misant sur la collaboration - et de favoriser les échanges et les consultations entre experts de la pharmacie hospitalière à l'échelle nationale.

Dans la salle d'exposition de la valorisation, deux thèmes illustratifs ont été disposés bien en vue sur des chevalets. D'abord, la $\mathrm{SCPH}$ a répondu à une invitation du Conseil consultatif sur la mise en œuvre d'un régime national d'assurance-médicaments lui demandant de recommander des membres de la Société qui pourraient parler avec confiance au sujet d'un régime d'assurance-médicaments et exposer la position de l'organisme au cours d'une série de tables rondes pancanadiennes pendant l'été. Parmi les 33 membres recommandés par la SCPH, 14 ont été invités par le Conseil consultatif à participer à neuf tables rondes. Le rapport final du Conseil consultatif est attendu pour le printemps 2019. Deuxièmement, la SCPH a continué de communiquer à Santé Canada les préoccupations de ses membres concernant la déclaration obligatoire des réactions indésirables graves aux médicaments et des incidents relatifs aux instruments médicaux par les hôpitaux. Ce travail de valorisation nécessitait : de formuler des remarques sur les modifications proposées au Règlement sur les aliments et drogues du Canada et l'analyse des coûts et avantages complémentaire; de participer avec l'A.P.E.S., à la demande de Santé Canada en deux occasions, à des discussions privées sur le projet de règlement et sur les étapes suivantes menant à sa promulgation; et de participer au Groupe consultatif sur la sensibilisation, l'éducation et la rétroaction.

Dans la galerie sont aussi présentées les réponses de la SCPH aux demandes de consultation des parties prenantes suivantes : Santé Canada, au sujet du projet de règlement sur la surveillance de l'aide médicale à mourir, sur le renouvellement du Programme d'accès spécial et sur l'avis d'intention de restreindre le marketing et la publicité des opiö̈des ainsi que l'analyse des coûts-avantages subséquente de la proposition connexe; et HSO (Health Standards Organization en anglais, c.-à-d. l'Organisation de 
normes en santé) quant à la norme sur la Gestion des médicaments.

La SCPH a ajouté plusieurs éléments précieux à sa collection : la Synthèse canadienne sur l'optimisation de la médication (c.-à-d. Canadian Medication Optimization Briefing en anglais) intitulée Safe Transitions of Care for Patients Taking Opioids (https:// www.cshp.ca/canadian-medication-optimization-briefing-0), le Rapport sur les pharmacies hospitalières canadiennes 2016-2017 (www.hospitalpharmacysurvey.ca) et les Lignes directrices sur la prestation de services de télépharmacie (https:// www.cshp.cal guidelines). Les artistes en résidence de la SCPH mettent aussi la touche finale à une œuvre monumentale, Controlled Drugs and Substances in Hospitals and Healthcare Facilities: Guidelines on Secure Management and Diversion Prevention, qui sera en présentation libre d'ici peu. De plus, la SCPH et d'autres organismes de soins de santé ont concouru au projet du Collège royal des médecins et chirurgiens du Canada afin de créer un livre numérique intitulé Naviguer les urgences médicales: Un guide interactif sur la prise en charge du patient (https://www.cshp.ca/ clinical-pharmacy); Hospital News a collaboré avec la SCPH dans le but d'illustrer la pratique de la pharmacie hospitalière dans son numéro de juin 2018 (https://issuu.com/hospitalnews/docs/ hospital_news_june_2018_edition); et Elsevier - une entreprise internationale d'analyse de l'information spécialisée en science et en santé - a passé commande à la SCPH du chapitre peignant "Hospital Pharmacy Practice: A Case Study from Canada " dans l'édition originale de l'Encyclopedia of Pharmacy Practice and Clinical Pharmacy, qui sera publiée en ligne en juin 2019 (https://www.elsevier.com/books/encyclopedia-of-pharmacy-practiceand-clinical-pharmacy/babar/978-0-12-812735-3).

Les galeries d'art et la SCPH ont toutes deux adopté la technologie pour des buts semblables : les premières afin d'engager les visiteurs et d'enrichir leur expérience de l'art et la seconde afin de mieux engager ses membres et d'enrichir leur expérience au sein de l'organisme. Au cours de la dernière année, la SCPH a mis à jour son système de gestion d'association, le logiciel sur lequel s'appuie la prestation des services aux membres. Grâce à l'interface du système avec le portail amélioré des membres sur le site Web de la SCPH, les membres peuvent plus facilement gérer leur profil en ligne, interagir et faire affaire avec l'organisme, et communiquer avec leurs pairs.

La SCPH a continué de prospérer sous le regard vigilant des commissaires d'exposition à son emploi. Chacune de ces 15 employées dévouées méritent des éloges publics, mais je réserve ici une pensée particulière à seules quelques-unes d'entre elles en 2018. Plusieurs anniversaires d'embauche marquants ont été célébrés cette année : 5 ans pour Susan Maslin (agente de la planification des événements et des réseaux de spécialistes en pharmacie), 10 ans pour Cathy Lyder (directrice, secteur des membres et des programmes), 15 ans pour Janet Lett (agente de la Fondation de la SCPH) et 25 ans pour Gloria Day (agente du Conseil canadien de la résidence en pharmacie). De plus, Laurie Frid (agente générale de programmes) et Amanda Iannaccio (agente des publications) ont été accueillies de nouveau parmi nous : Laurie après une absence autorisée et Amanda après son congé de maternité et son congé parental. Enfin, Christopher Doody (agent intérimaire des publications) et Olga Chrzanowska (agente du Web pendant près de 10 ans) ont eu droit à des adieux chaleureux.

Pour ce qui est de la rétrospective des 15 dernières années, afin de faire mentir l'expression " une image vaut mille mots ", je vous laisse des centaines de mots qui esquissent une image impressionniste : un nuage de mots-clés tirés de l'ensemble de mes rapports de directrice générale.

Veuillez recevoir ma reconnaissance éternelle.

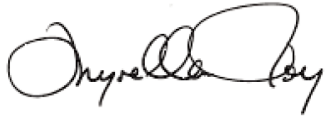

Myrella Roy, B. Sc. Phm., Pharm. D., FCCP

Ancienne directrice générale (de 2003 à 2018)

Société canadienne des pharmaciens d'hôpitaux 